\title{
X-ray tomographic microscopy at TOMCAT
}

\author{
F. Marone ${ }^{1}$, C. Hintermüller ${ }^{1}$, S. McDonald ${ }^{1,2}$, R. Abela ${ }^{1}$, \\ G. Mikuljan ${ }^{1}$, A. Isenegger ${ }^{1}$ and M. Stampanoni ${ }^{1,3}$ \\ ${ }^{1}$ Swiss Light Source, Paul Scherrer Institut, CH-5232 Villigen, Switzerland \\ ${ }^{2}$ Department of Radiology, University of Lausanne Medical School, Lausanne, Switzerland \\ ${ }^{3}$ Institute for Biomedical Engineering, University and ETH Zurich, 8092 Zurich, Switzerland
}

\begin{abstract}
Synchrotron-based X-ray Tomographic Microscopy is a powerful technique for fast, non-destructive, high resolution quantitative volumetric investigations on diverse samples. At the TOMCAT (TOmographic Microscopy and Coherent radiology experimenTs) beamline at the Swiss Light Source (SLS), synchrotron light is delivered by a 2.9 T superbend. The main optical component, a Double Crystal Multilayer Monochromator, covers an energy range between 8 and 45 $\mathrm{keV}$. The standard TOMCAT detector offers field of views ranging from $0.75 \times 0.75 \mathrm{~mm}^{2}$ up to $12.1 \times 12.1 \mathrm{~mm}^{2}$ with a theoretical resolution of $0.37 \mu \mathrm{m}$ and $5.92 \mu \mathrm{m}$, respectively. The beamline design and flexible endstation setup make a large range of investigations possible. In addition to routine measurements, which exploit the absorption contrast, the high coherence of the source also enables phase contrast tomography, implemented with two complementary techniques. Differential Phase Contrast (DPC) imaging has been fully integrated in terms of fast acquisition and data reconstruction. Scans of samples within an aqueous environment are also feasible. The second phase contrast method is a Modified Transport of Intensity approach that yields a good approximation of the 3D phase distribution of a weakly absorbing object from a single tomographic dataset. Typical acquisition times for a tomogram are in the order of few minutes, ensuring high throughput and allowing for semi-dynamical investigations and in-situ experiments. Raw data are automatically post-processed online and full reconstructed volumes are available shortly after a scan with minimal user intervention. In addition to a beamline overview, a selection of high-impact tomographic applications will be presented.
\end{abstract}

Keywords: X-ray imaging, synchrotron microtomography, Differential Phase Contrast, Transport of Intensity, fast reconstruction algorithms.

\section{INTRODUCTION}

$\mathrm{X}$-ray tomographic microscopy is a powerful technique for fast non-destructive, high resolution, quantitative volumetric investigations on an ample variety of samples, with application ranging from the life sciences through materials science to environmental and earth sciences. Recent developments of microtomographic devices at third generation synchrotron facilities all over the world have made volumetric information of specimens at the micrometer and sub-micrometer scale routinely accessible ${ }^{1-4}$. Absorption based and "edge-enhanced" tomographic experiments have been performed at TOMCAT $^{5}$ since its kick off in June 2006 and a large number of cutting edge scientific results in a variety of subjects ${ }^{6}$ has been achieved.

Interest in phase contrast imaging has also been considerably growing during the past few years. In fact exploitation of the coherent nature of synchrotron radiation has evident advantages, in particular for biological samples, typically showing a rather weak absorption contrast at hard X-ray energies, if not ad-hoc prepared (e.g. casting and staining) as currently required by most high resolution imaging techniques. Phase contrast tomography gives fast access to 3D structural information in near-native biological samples without need for time consuming tissue alteration/modification for contrast enhancement. In addition, phase signals can be produced with much lower dose deposition compared to absorption, an important point when radiation damage is an issue. Not only the life sciences benefit from phase contrast tomography. Studies on new materials and fibers, mostly relying on light elements such as carbon, also increasingly exploit this technique.

Developments in X-Ray Tomography VI, edited by Stuart R. Stock,

Proc. of SPIE Vol. 7078, 707822, (2008) · 0277-786X/08/\$18 - doi: 10.1117/12.803147

Proc. of SPIE Vol. 7078 707822-1 
At TOMCAT we also perform phase contrast tomography. We offer to the user community two complementary techniques: Modified Transport of Intensity approach ${ }^{7}$ and Differential Phase Contrast method (DPC) ${ }^{8}$. The first phase contrast approach yields a good approximation of the 3D phase distribution of a weakly absorbing object from a single tomographic dataset without need of additional hardware. It is particularly suited for small specimen when high resolution is required. This method is fully integrated at the TOMCAT beamline and is increasingly requested by the user community: the first high quality and high impact results have been recently published ${ }^{6}$. The second phase contrast technique available at TOMCAT, the Differential Phase Contrast (DPC) method $^{8}$ is characterized by a higher sensitivity by moderate resolution and gives the best results with larger samples. During the past year, considerable efforts have been spent on fully integrating this approach, both in terms of fast acquisition and reconstruction, at TOMCAT. Since recently available to the user community, this new feature makes the beamline unique in the world.

In this paper, after a brief overview of the beamline, we present the recent hardware and software advancements regarding the implementation of the DPC technique at TOMCAT. In addition, we illustrate our general solution in terms of data acquisition protocol, postprocessing and reconstruction, which has been optimized for speed, enabling high throughput experiments. Finally we discuss a selection of high impact applications and briefly introduce our future projects.

\section{BEAMLINE OVERVIEW}

\subsection{Front End}

The TOMCAT beamline ${ }^{5}$ at the SLS (a medium energy machine with $2.4 \mathrm{GeV}$ and stable top up at $400 \mathrm{~mA}$ ) has been in user operation for about 2 years. Synchrotron light is delivered to the beamline by a $2.9 \mathrm{~T}$ superbend, with a critical energy of $11.1 \mathrm{keV}$, which ensures considerable flux at energies $>20 \mathrm{keV}$ allowing experiments in a larger spectrum of the hard X-rays. In terms of optics, TOMCAT, being an imaging beamline, has been kept simple, to prevent degradation of the beam profile, stability and coherence. In this way, optimal background corrections are guaranteed and phase contrast imaging is possible. The only optical elements present are a Chemical Vapor Deposited (CVD) diamond window, separating the UHV sector of the machine from the HV of the beamline and a fixed-exit double crystal multilayer monochromator (DCMM). The CVD window has been preferred to standard Be-windows, because its smaller surface roughness is imperative for coherence preservation. The DCMM monochromator covers an energy range from 8 to $45 \mathrm{keV}$. It is located at approximately $7 \mathrm{~m}$ from the source in the front end. This unusual choice has been dictated by the need to extract the largest beam out of the fixed beamline length.

\subsection{Standard endstation setup}

The TOMCAT endstation, with its simple design, allows a large degree of flexibility, making a variety of experiments possible. In its standard setup (Figure 1), the TOMCAT instrument features the beam conditioner block, the sample manipulator unit and the microscope. The beam conditioner consists of a mechanical-magnetic millisecond shutter, a JJslits pair and an ionization chamber. The PSI designed and manifactured sample manipulator guarantees high reproducibility (about $0.1 \mu \mathrm{m}$ ) for sample centering and acquisition of reference images; the air-bearing rotation axis performs with a wobble $<2.5 \mu \mathrm{rad}$. The standard TOMCAT microscope is based on a diffraction limited optics. X-rays converted to visible light by a scintillator are magnified with interchangeable (motorized) objectives. Field of views range from $0.75 \times 0.75 \mathrm{~mm}^{2}$ up to $12.1 \times 12.1 \mathrm{~mm}^{2}$ with a theoretical pixel size of $0.37 \mu \mathrm{m}$ and $5.92 \mu \mathrm{m}$, respectively. A more efficient second system, based on a high-aperture tandem 1:1 configuration and accepting a diagonal up to $40 \mathrm{~mm}$, is also available for large field of view experiments which require only moderate resolution. The CCD camera has a $2048 \times 2048$ pixels chip $\left(7.4 \times 7.4 \mu \mathrm{m}^{2}\right.$ pitch) and an internal RAM memory (camRAM) of $4 \mathrm{~Gb}$, where acquired images are transferred at very high rates (up to $14.7 \mathrm{fps}$ at full frame). Depending on the selected operating mode, image data are kept in the camRAM or are transmitted via a IEEE 1394a Firewire connection to the Windows PC used to control the camera.

This standard setup is mostly used for routine absorption experiments. The high coherence of the source is, however, also increasingly exploited for phase contrast tomography implemented at TOMCAT with two complementary 
techniques. This standard endstation configuration is also used for one of these phase contrast methods, a Modified Transport of Intensity ${ }^{7}$ approach that yields a good approximation of the 3D phase distribution of a weakly absorbing object, from a single tomographic dataset acquired with a sample-detector distance of few centimeters.

The generous space around the sample holder and sample stage easily permits installation of equipment for in-situ tomographic experiments (see section 4.2). Use of a cryojets, furnaces, electrochemical and compression/tensile devices at TOMCAT are common.

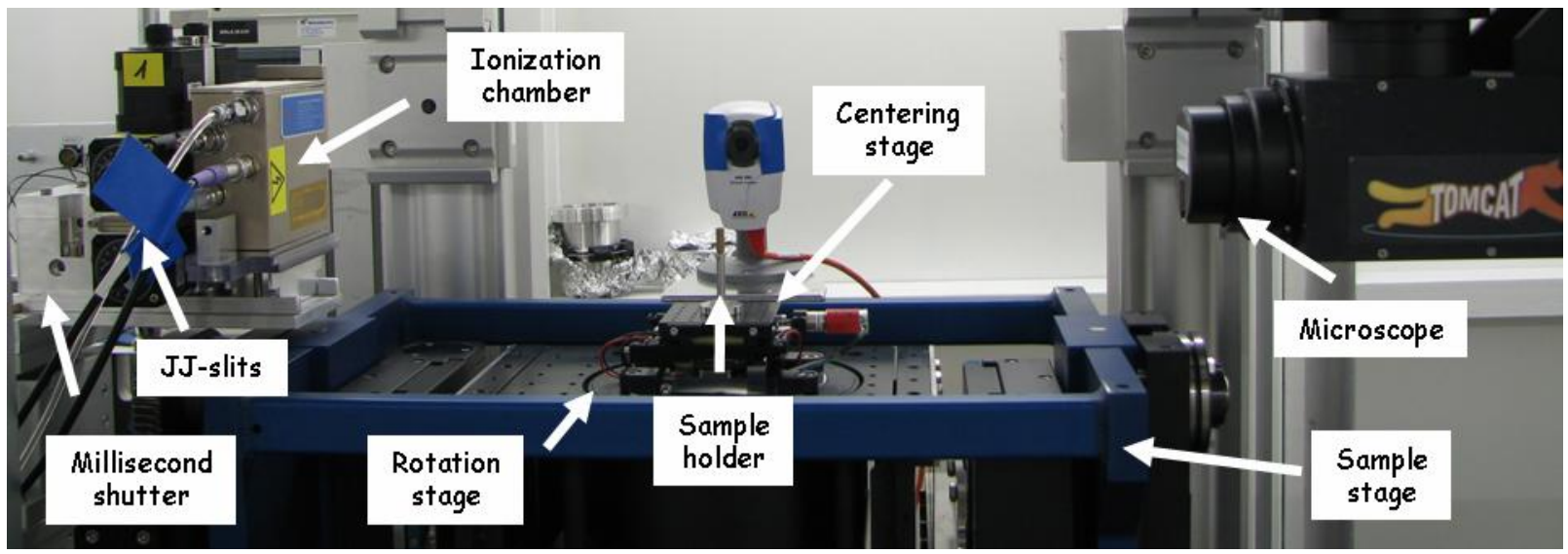

Figure 1. Standard setup of the TOMCAT endstation.

\subsection{Endstation setup for DPC experiments}

For the second phase contrast technique available at TOMCAT, the DPC method ${ }^{8}$, ad-hoc hardware is required (Figure 2). Our solution ${ }^{9}$ can be quickly plugged in on the standard endstation setup. The hard X-ray interferometer works with 2 gratings, a phase grating which splits the incoming beam and an absorption grating, which works as an analyzer, positioned at uneven Talbot distances, where the diffracted beam produces an interference pattern. The silicon phase grating has a pitch of $4 \mu \mathrm{m}$, twice that of the gold analyzer grating. A phase stepping approach is used to transform the local fringes position of the interference pattern into signal intensity variations, containing information on the phase gradient of the object. The 2 gratings are mounted on a massive support completely decoupled from the sample stage to avoid vibrations during sample rotation and translation. While the relative distance between the 2 gratings needs to be adjusted manually, the assembly is motorized along 3 directions. The possibility to remotely displace the gratings along the beam direction is particularly useful for setup adjustments. For the phase stepping approach, one of the gratings needs to be displaced transversely to the incoming beam over one period $(2 \mu \mathrm{m})$ in at least 4-5 steps where radiographs are acquired. This translation has to be accurate, fast and reproducible. At TOMCAT, we solved this issue in 2 different ways: with an in-house developed nanoconverter and/or a piezo stage (Figure 2).

For the best quality results, it is mandatory to avoid large phase jumps, in particular at the sample surface. For this reason, for most experiments phase matching is necessary and the sample needs to be scanned while in a wet environment. For this purpose we developed an aquarium ${ }^{9}$, consisting of an aluminum tank with entrance and exit Kapton windows along the beam path. The sample holder comes into the aquarium through a hole at its bottom. Specimens, glued on supports, are fixed to the sample holder via a magnetic mechanism. A gasket system at the bottom of the tank ensures smooth sample rotation, translation for reference acquisition and centering, while the aquarium chamber remains still. Immobility of the aquarium is important to avoid changes in the flat part of the radiographs, since the Kapton windows always remain in the beam path. For removing possible air bubbles present in the phase matching liquid before the start of the scan, a small vibrator can be connected to the aquarium. In addition, the tank can also be closed and slightly evacuated to facilitate degassing. Air bubbles are, however, mostly avoided if viscous liquids, such as liquid paraffin, are used. In contrast, bubble formation in aqueous environment during a scan is more difficult to prevent, possibly because of beam damage. 
Adequate sample preparation is also significantly contributing to successful results and in particular to an optimal contrast in the reconstructions. In our experience, the best images have been obtained for samples embedded in paraffin, where particular care has been taken to avoid air inclusions, responsible for strong artifacts in the reconstructions. Tomograms of samples in natural state are, however, also possible.

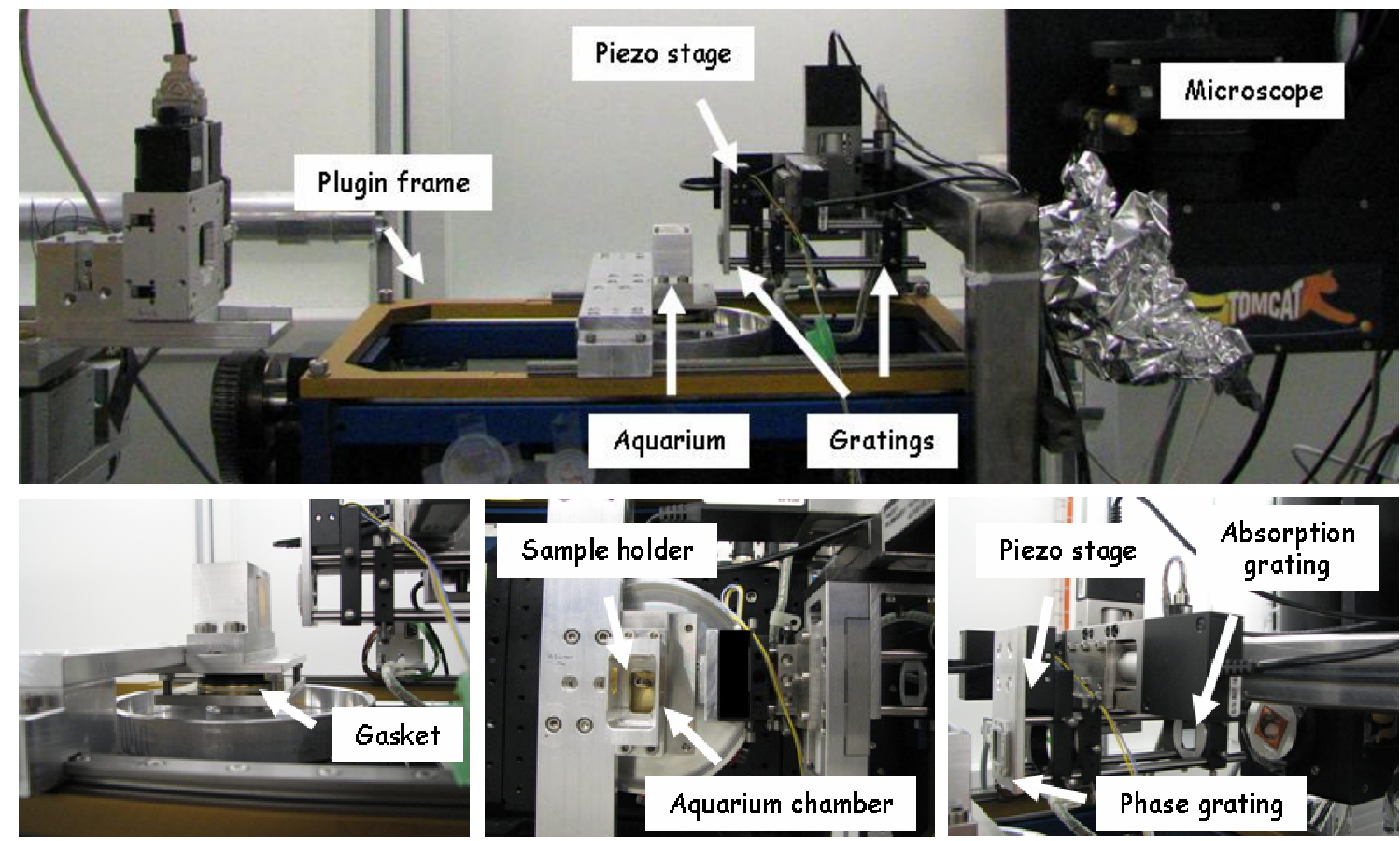

Figure 2. Setup of the TOMCAT endstation for DPC experiments. Top: overview; Bottom: left: detail of the gasket system allowing rotation, translation and centering of a specimen in the aquarium chamber - center: top view of the aquarium - right: detail of the gratings assembly.

\section{SOFTWARE}

\subsection{Fast data acquisition}

In addition to the improved photon flux delivered by the superbend, fast acquisition of high quality data at TOMCAT is achieved by the combination of a continuous scanning protocol with an optimized acquisition software, where the sample rotates continuously over a 180 degrees range, while images are simultaneously collected by the camera. The rotation speed is adapted according to the frame rate, which depends on the chosen camera settings (e.g. binning and image size). If the image space is adequately sampled, resolution and sharpness of the imaged specimens are guaranteed. This continuous rotation does not only increase the acquisition speed, but it also helps reducing typical local tomography artifacts. In fact, if the rotation speed is correctly optimized, the part of the sample located outside the field of view covers more than one pixel during the acquisition of one image, resulting blurred and therefore contributing with less information to the reconstructed image. The optimized acquisition software ${ }^{10}$ implemented at TOMCAT exploits particular properties of the detector: the $4 \mathrm{~Gb}$ internal RAM memory and the First In First Out (FIFO) buffer acquisition mode. Making use of these properties, the camera records and stores images while simultaneously transmitting previously acquired data, temporarily stored in the camRAM, to the file server. This approach eliminates most dead time and almost reduces the scan time to only the total exposure time.

The same approach with continuous sample rotation and FIFO acquisition mode is also used for DPC experiments, where a complete continuous scan is acquired for each phase step. This solution considerably decreases the total 
acquisition time, as opposed to a more traditional static approach, where for each angle the rotation stage stops and projections for the required phase steps are taken one after the other. The increased speed does not impair results quality. Our new acquisition scheme makes high throughput phase contrast studies possible.

\subsection{Online post-processing}

During acquisition, a selection of sinograms is computed on the fly, allowing quality check of the recorded tomogram right after the end of a scan. A user friendly web based application ${ }^{10}$ (Figure 3) simplifies this task. This application is also used to select and fine tune the parameters (e.g. center of rotation and filter) which will be subsequently used for the reconstruction of the entire data set (see section 3.3). Routines for removing ring artifacts and converting the results to TIFF images can also be selected.

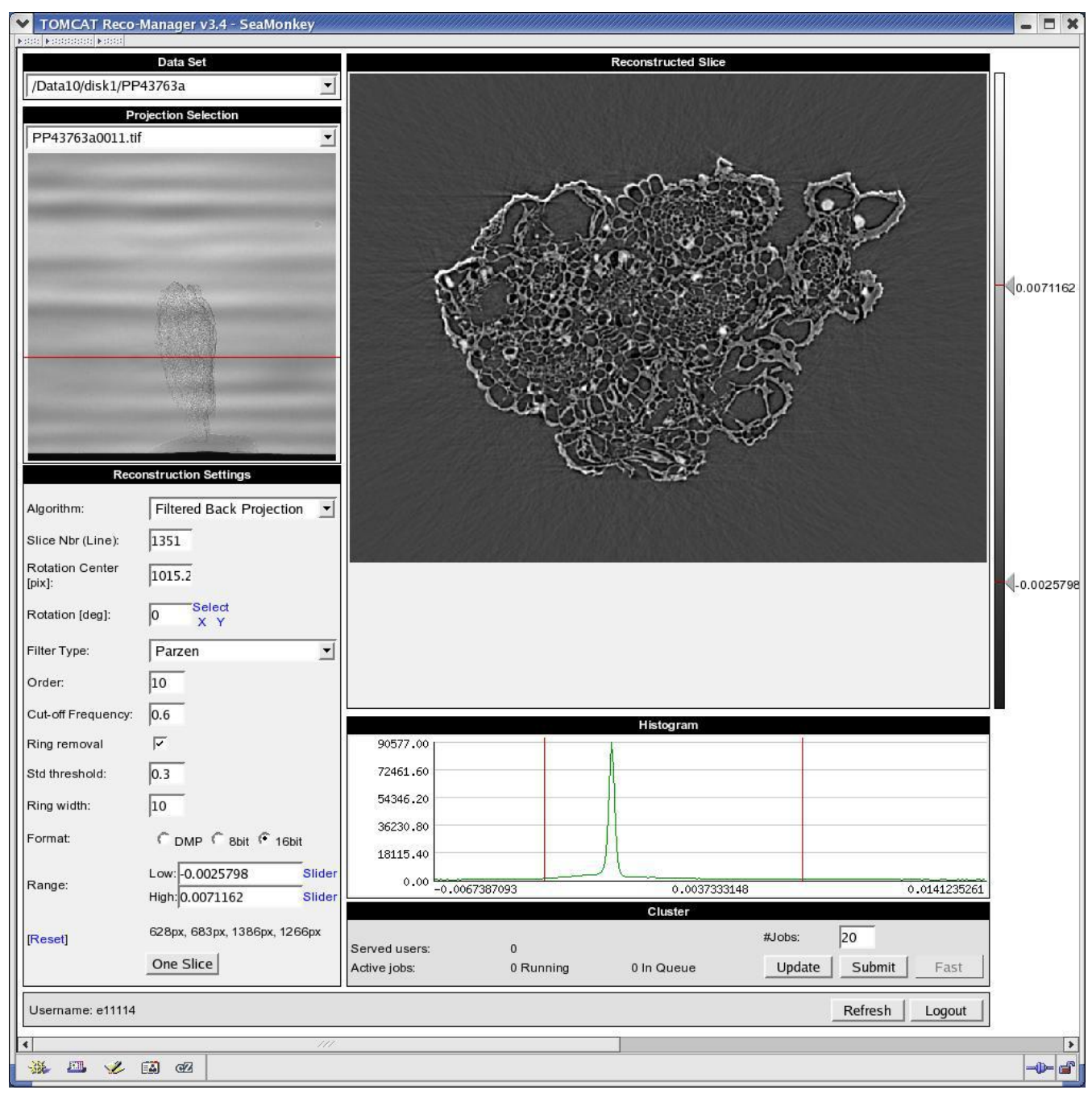

Figure 3. Web based application used to perform quick quality checks of the acquired tomograms right after the end of a scan. This user friendly application is also helpful to select and fine tune the reconstruction parameters (on the left panel, e.g. algorithm, rotation center, filter and region of interest) using a pool of sinograms. Routines for removal of ring artifacts and format conversion of the resulting slices can also be applied. Adjustment of the image contrast according to the histogram and fast zoom-in/zoom-out are also features of this application. After selection of the optimal parameters, the reconstruction of the entire dataset can be submitted to our Linux PC cluster with the "Submit" button and the reconstruction progress can be monitored. 


\subsection{Reconstructions}

The complete reconstruction of tomographic datasets, from the raw projections to the 3D volume, occurs on a 5 node Linux cluster, where each node is equipped with 2 dual core Xeon processors clocked at $3.0 \mathrm{GHz}$. Such job can be submitted to the cluster directly from the web application (Figure 3), with just one mouse click. The reconstruction load is divided and balanced among the available computing units by DICAT (Distributed Computing Application for Tomography), an in-house developed Java based two-tier client/server application ${ }^{10}$. Each node first computes a fraction of the sinograms, which are stored locally. After completion of this first step, single slices are reconstructed and saved on the file server. At TOMCAT two different reconstruction algorithms are available ${ }^{11}$ : a routine based on the well established Filter Back-Projection approach and a faster code based on the Fourier Transform method ${ }^{12}$. Each single step of this reconstruction pipeline has been optimized for speed, to be able to fully exploit the advantages of our fast acquisition protocol. A high resolution $2048 \times 2048$ (1501 projections) dataset is reconstructed on the cluster in less than 12 minutes; reconstructions of binned tomograms are available few minutes after the end of a scan. This acquisition and reconstruction speed makes high throughput studies a reality. On going work is focusing on further optimization of the reconstruction process, in particular by exploiting the computation power and characteristics of emerging GPU technology.

\subsection{Post processing and reconstruction of DPC data}

Post processing and reconstruction of the acquired DPC data has been completely integrated in our standard tools developed for absorption tomography. In fact, our in-house developed code for the sinogram computation ${ }^{10}$ can also handle phase contrast projections and compute DPC sinograms. This step can, however, not be performed on the fly, because information from all phase steps are necessary for the calculation of one line in the sinogram. The code has, however, been highly optimized so that few minutes after the end of the scan, a selection of sinograms is available. In addition, an imaginary filter ${ }^{13}$ has been integrated in our Filter Back-Projection algorithm, so that direct reconstruction of the index of refraction of the object from DPC sinograms is possible without need for integration to the phase. The entire reconstruction process is implemented on the cluster and a high quality tomographic phase dataset (1000 projections, 9 phase steps) can be acquired and reconstructed in less than one hour.

\section{APPLICATIONS}

\subsection{Phase contrast tomography}

Unique fossil charcoalified flowers, showing very little absorption in the hard X-ray regime, have been successfully imaged with the Modified Transport of Intensity approach ${ }^{7}$, requiring the acquisition of one single dataset with the sample at an optimal distance from the detector. The high resolution of this technique and the top quality images obtained (Figure 4) allowed the disclosure of internal details of this unique fossil specimen, which revolutionized the phylogeny of the first flowering plants and revived an early strongly debated hypothesis ${ }^{6}$. Such scientific results would have not been otherwise possible without sample destruction.

Soft biological tissues in near-natural state benefit from the higher sensitivity of the DPC approach. Figure 5 shows an example of what can be achieved with this technique. This mouse brain has been embedded in paraffin, but no contrast agent has been added. The excellent contrast allows the visualization for instance of the cortex and the grey and white matter in the cerebellum. Even small blood vessels are visible.

The different sensitivity and resolution achieved with the two phase contrast imaging approaches implemented at TOMCAT is illustrated in Figure 6. Two tomograms of the same post-transplant human myocardium biopsy have been acquired with the two techniques and the same coronal slice is shown. The higher sensitivity of the DPC method and the higher resolution achieved with the Modified Transport of Intensity approach are clear. The resolution of the DPC method depends on the Talbot distance and is limited by the grating pitch ${ }^{8}$, while the field of view could, in theory, be as large as the available beam. In contrast, for the propagation method ${ }^{7}$, Fresnel fringes need to be detected and therefore high resolution radiographs and as a consequence field of views in the order of 1-2 mm are imperative. The best results are obtained with a $10 \mathrm{x}$ magnification objective, a $1.5 \times 1.5 \mathrm{~mm}^{2}$ field of view and a $0.74 \times 0.74 \mathrm{~mm}^{2}$ pixel size. Higher 
magnifications do not bring the expected increase in resolution, because this is theoretically limited to $\sqrt{d \cdot \lambda}$, where $\mathrm{d}$ is the sample-detector distance and $\lambda$ is the used wavelength.

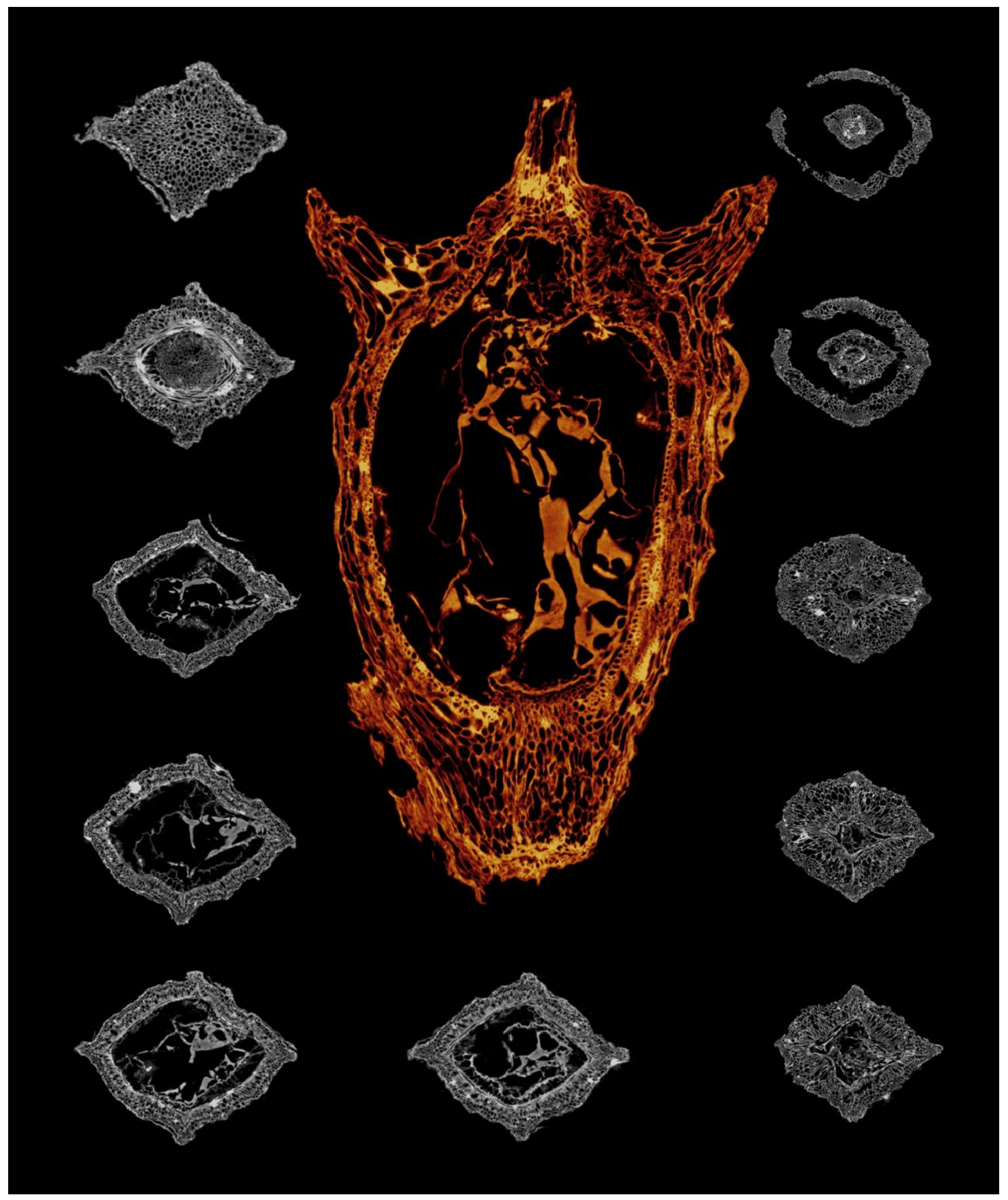

Figure 4. Axial (small, at the sides) and longitudinal (large, central) cross sections through the 3D reconstruction of the phase information of a $120 \mathrm{Ma}$ old charcoalified fossil seed. The specimen width is about $1 \mathrm{~mm}(0.7 \mu \mathrm{m}$ pixel size, 1500 projections, 20 $\mathrm{keV})$. 


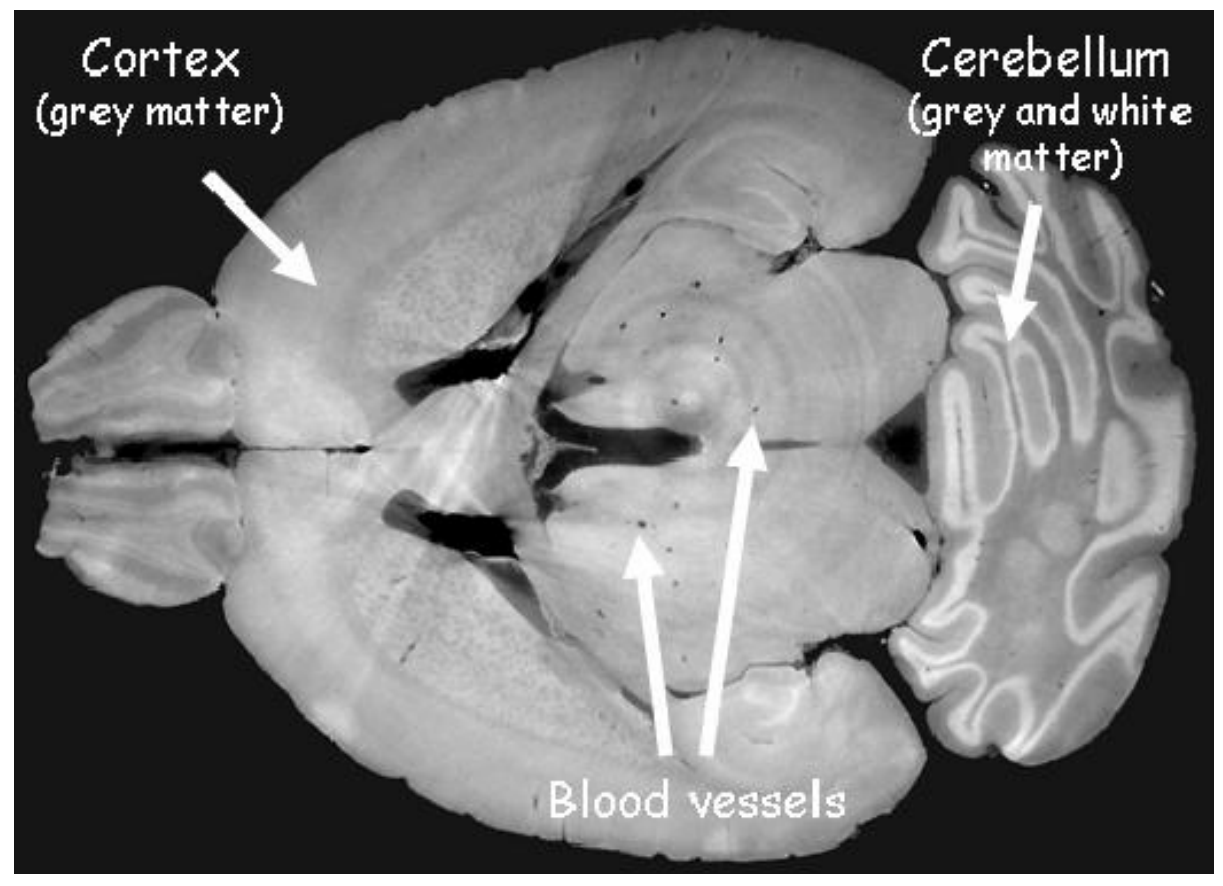

Figure 5. Axial slice through the $3 \mathrm{D}$ reconstruction of the phase information of a native mouse brain embedded in paraffin $(10 \mu \mathrm{m}$ pixel size, 1000 projections, $25 \mathrm{keV}$, 3rd Talbot distance, 9 phase steps). Blood vessels, grey and white matter are clearly visible without need for a contrast agent.
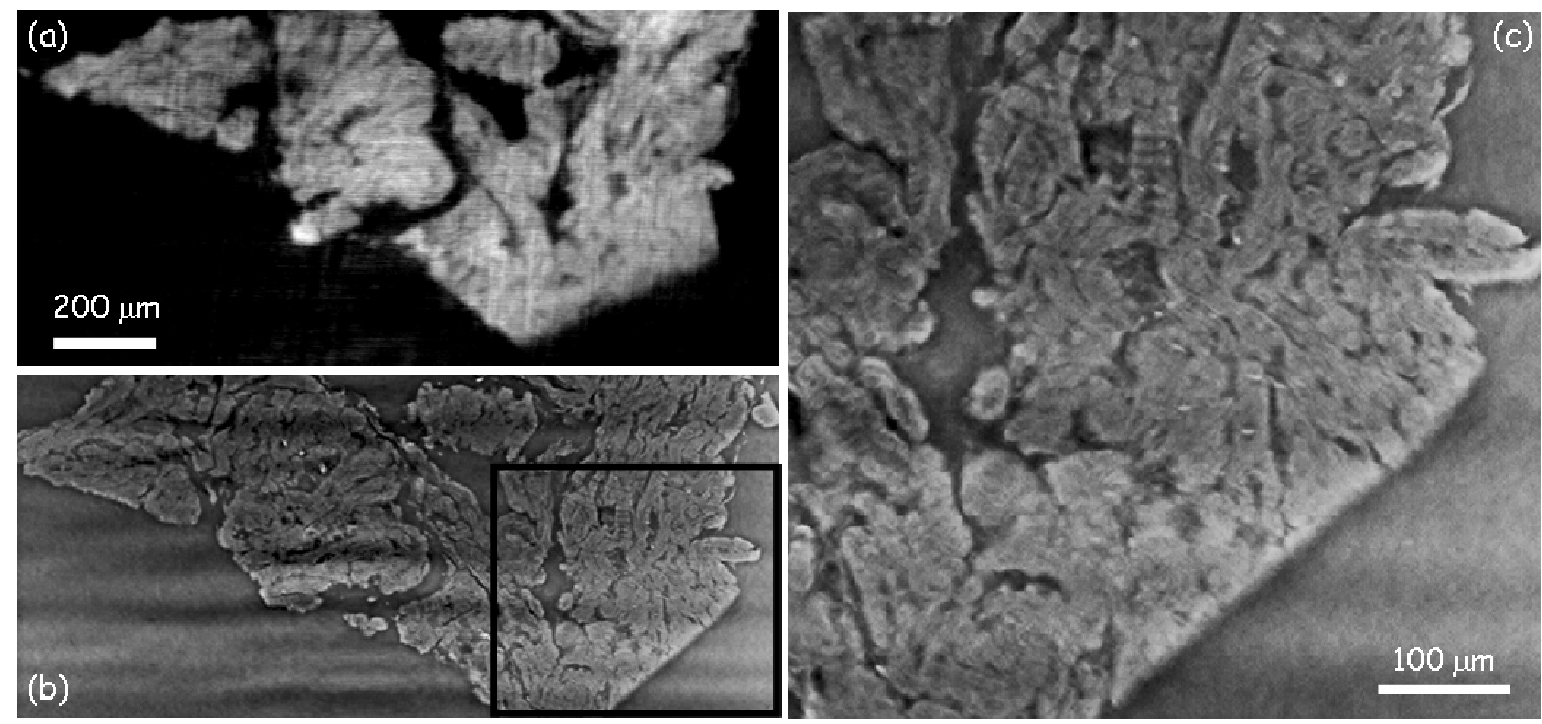

Figure 6. Comparison of two tomograms of a post-transplant human myocardium, embedded in solid paraffin (Sample courtesy of Dr. S. De Marchi, Inselspital, Bern, Switzerland and Dr. M. Gugger, Pathologisches Institut, University of Bern, Switzerland) obtained with the two phase contrast techniques implemented at TOMCAT - (a) DPC tomogram (1.4 $\mu \mathrm{m}$ pixel size, 500 projections, $25 \mathrm{keV}$, 3rd Talbot distance, 9 phase steps). (b) Tomogram acquired and reconstructed using the Modified Transport of Intensity approach (0.7 $\mu \mathrm{m}$ pixel size, 1500 projections, $20 \mathrm{keV}$ ). (c) Magnification of the region enclosed in the black box in (b). 


\subsection{In-situ semi-dynamical absoption contrast tomography}

Localized corrosion has a large technological impact on areas ranging from the performance of alloys for biomedical applications to the long term storage of nuclear waste. Corrosion pits are, however, very difficult to study as the location and time of initiation is extremely hard to predict. With fast X-ray absorption contrast microtomography and total scan times in the order of few minutes, it is possible to follow the evolution of corrosion pit at the flat top of a metal pin in solution. In this experiment (Figure 7), electrochemical control was provided by an ad-hoc designed microcapillary cell $^{14}$, so that for the first time the 3D evolution of single pits could be directly observed in-situ and the dynamics of pit development as a function of applied potential and solution composition could be studied.
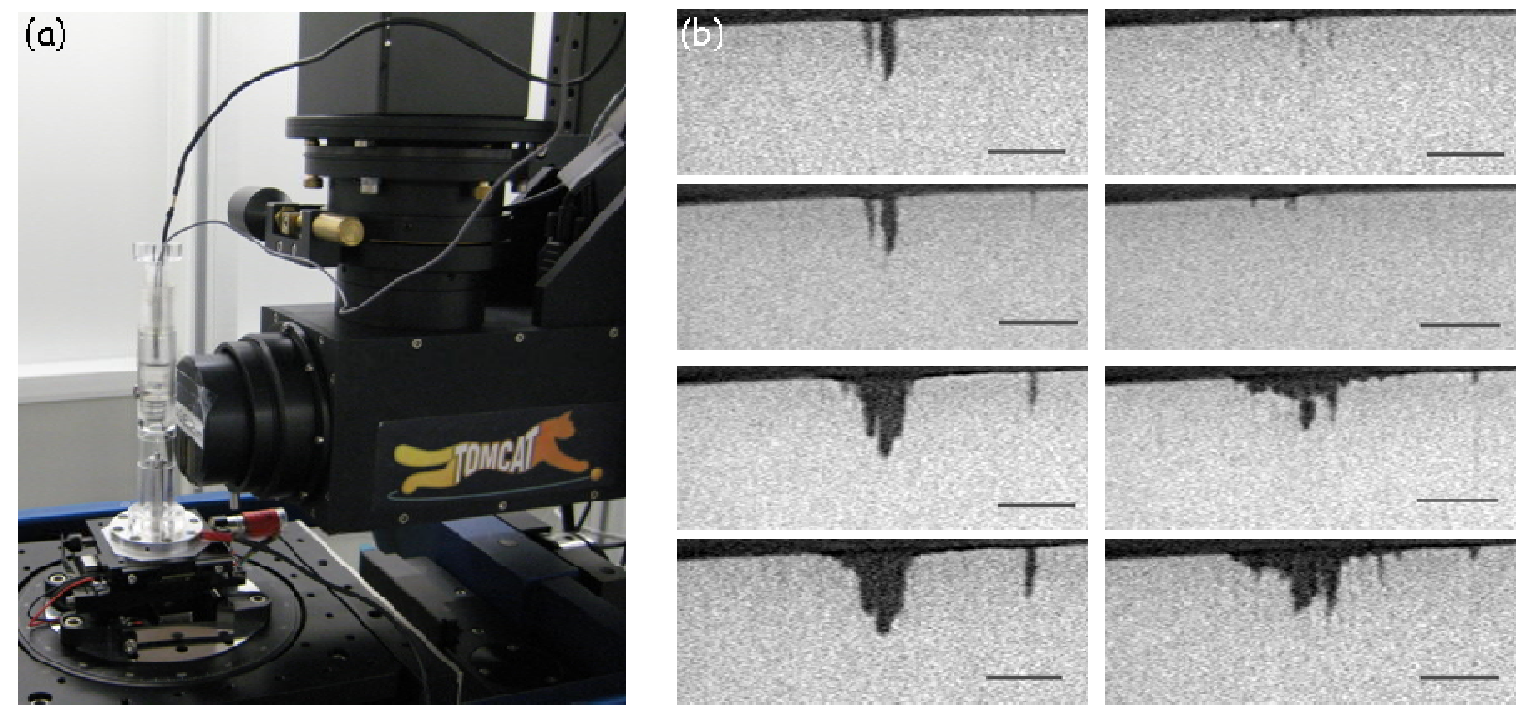

Figure 7. (a) Endstation setup for in-situ corrosion tomographic experiments. An ad-hoc designed microcapillary electrochemical cell $^{14}$ is mounted on the standard centering stage. (b) Two different vertical cross-sections through the 3D reconstruction of a 303 stainless steel pin. From the top to the bottom, corrosion evolution as a function of time and increased applied current is observed. Scale bar: $20 \mu \mathrm{m}(0.7 \mu \mathrm{m}$ pixel size, 500 projections, $40 \mathrm{keV}, 5$ minutes scan time) (Image courtesy: A. Davenport, University of Birmingham, Birmingham, England).

\section{OUTLOOK}

\subsection{Nano-tomography}

While the TOMCAT beamline routinely offers tomographic capabilities in the micrometer range exploiting both absorption and phase contrast, voxel sizes as small as $100 \mathrm{~nm}$ are the current challenge and are reached only by few hard $\mathrm{X}$-ray instruments around the world. To fill this gap, recent work focused on the submicrometer regime, with the aim of expanding the 3D spatial resolution range accessible at TOMCAT down to $100 \mathrm{~nm}$. In particular, we developed and implemented a full field, hard X-ray microscope reaching a theoretical voxel size of $30 \mathrm{~nm}$ and a field of view of $50 \mu \mathrm{m}$. Additional characteristics of this new instrument are stability, operation ease and user-friendliness, mandatory requirements to be able to offer this new endstation with its $3 \mathrm{D}$ spatial resolution in the order of $100 \mathrm{~nm}$ to the user community. This new experimental setup consists of a beam-shaping condenser lens ${ }^{15}$, which produces a square-shaped flat-top illumination and a magnifying Fresnel zone plate ${ }^{16}$. It is presented in details in this proceeding ${ }^{17}$, in addition to radiographic examples and tomographic reconstructions with a voxel size of $50 \mathrm{~nm}$ of simple and more complex absorbing objects. Our most recent experiments also show that edge-enhancement can be exploited to image phase objects. 


\subsection{Ultra-fast tomography}

We recently started the design of an ultra-fast tomographic endstation at TOMCAT, with the goal to obtain full 3D tomograms at a frequency of $0.1-1 \mathrm{~Hz}$, with resolution in the micron scale. The access to subsecond, fully $3 \mathrm{D}$, nondestructive quantitative imaging, will open completely new horizons in different application fields including medicine, biology and materials science.

\section{ACKNOLEDGMENTS}

S. McDonald was supported by the Centre d'Imagerie Biomédicale (CIBM) of the UNIL, EPFL, UNIGE, CHUV and HUG. We thank F. Pfeiffer for valuable discussion, C. David for the fabrication of the x-ray optics and O. Bunk for the implementation of the piezo stage.

\section{REFERENCES}

1. Beckmann, F., Vollbrandt, J., Donath, T., Schmitz, H.W. and Schreyer, A., "Neutron and synchrotron radiation tomography: New tools for materials science at the GKSS-Research Center", Nuclear Instruments and Methods in Physics Research Section A 542, 279-282, 2005.

2. Weitkamp, T., Raven, C. and Snigirev, A., "Imaging and microtomography facility at the ESRF beamline ID22", in Developments in X-Ray Tomography II, ed. U. Bonse, Proc. SPIE 3772, 311-317, 1999.

3. De Carlo, F., Xiao, X.H. and Tieman, B., "X-ray tomography system, automation and remote access at beamline 2BM of the Advanced Photon Source", in Developments In X-Ray Tomography V, ed, U. Bonse, Proc. SPIE 6318, K3180, 2006.

4. Uesugi, K., "Development of High Spatial Resolution X-Ray CT System at BL47XU in SPring-8", Nuclear Instruments and Methods in Physics Research Section A 467-468, 853-856, 2001.

5. Stampanoni, M., Groso, A., Isenegger, A., Mikuljan, G., Chen, Q., Bertrand, A., Henein, S., Betemps, R., Frommherz, U., Böhler, P., Meister, D., Lange, M. and Abela, R., "Trends in synchrotron-based tomographic imaging: the SLS experience", in Developments in X-Ray Tomography V, ed. U. Bonse, Proc. SPIE 6318, 63180M1$14,2006$.

6. Friis E.M., Crane P.R., Pedersen K.R., Bengtson S., Donoghue P.C.J., Grimm G.W. and Stampanoni M., "Phasecontrast X-ray microtomography links Cretaceous seeds with Gnetales and Bennettitales", Nature 450, 549-552, 2007.

7. Grošo, A., Abela, R. and Stampanoni, M., "Implementation of a fast method for high resolution phase contrast tomography", Optics Express 14, 8103-8110, 2006.

8. Weitkamp, T., Diaz, A., David, C., Pfeiffer, F., Stampanoni, M., Cloetens, P. and Ziegler, E., "X-ray phase imaging with a grating interferometer", Optics Express 12, 6296-6304, 2005.

9. McDonald, S., Marone, F. and Stampanoni, M., in preparation.

10. Hintermüller, C., Marone, F., Isenegger, A. and Stampanoni, M., in preparation.

11. Marone, F., Hintermüller, C., Geus, R. and Stampanoni, M., in preparation.

12. Down, B.A., Campbell, G.H., Marr, R.B., Nagarkar, V., Tipnis, S., Axe, L. and Siddons, D.P., "Developments in synchrotron x-ray computed microtomography at the National Synchrotron Light Source", in Developments in X-Ray Tomography II, ed. U. Bonse, Proc. SPIE 3772, 224-236, 1999.

13. Pfeiffer, F., Bunk, O., Kottler, C. and David, C., "Tomographic reconstruction of three dimensional objects from hard X-ray differential phase contrast projection images", Optics Express 15, 1175-1181, 2007.

14. Eckermann, F., Suter, T., Uggowitzer, P.J., Afseth, A., Stampanoni, M., Marone, F., Schmutz, P., "In-situ Microtomographically-monitored and Electrochemically-controlled Corrosion Initiation and Propagation in AlMgSi Alloy AA6016", submitted.

15. Jefimovs, K., Vila-Comamala, J., Stampanoni, M., Kaulich, B. and David, C., "Beam-shaping condenser lenses for full-field transmission X-ray microscopy", J. Synchrotron Rad. 15, 106-108, 2008.

16. Jefimovs, K., Bunk, O., Pfeiffer, F., Grolimund, D., van der Veen, J.F. and David, C., "Fabrication of Fresnel zone plates for hard X-rays", Microelectronic Engineering 84, 1467-1470, 2007. 
17. Stampanoni, M., Marone, F., Mikuljan, G., Jefimovs, K., Trtik, P., Vila-Comamala, J., David, C. and Abela, R., "Hierarchical, multimodal tomographic X-ray imaging at a superbend", this proceeding. 